\title{
Folded and confined one-dimensional plasmons in modulated wires
}

\author{
Florent Perez, Bernard Jusserand, Claus Dahl,* and Marcel Filoche \\ France Télécom, Centre National d'Etudes des Télécommunications, Paris B, Laboratoire de Bagneux, \\ 196 Avenue Henri Ravera, 92220 Bagneux, France \\ Laurence Ferlazzo-Manin and Bernard Etienne \\ Laboratoire de Microstructures et Microélectronique, Centre National de la Recherche Scientifique, \\ 196 Avenue Henri Ravera, 92220 Bagneux, France
}

(Received 22 April 1996)

\begin{abstract}
We demonstrate theoretically and experimentally that one-dimensional plasmons confined in doped wires are either extended or longitudinally confined when the lateral width of the wires is periodically modulated. Theoretical dispersion curves, calculated within a classical framework, reproduce well the experimental dispersion deduced from Raman scattering on deep etched modulated wires. Zone-edge gap openings, increasing with the modulation amplitude, are exhibited. We show that deep etching allows good control of complex geometries and provides quasiabrupt boundary conditions. [S0163-1829(96)50140-2]
\end{abstract}

Advances in the microfabrication techniques of semiconductors ${ }^{1}$ have given access to devices with onedimensional (1D) confinement of electrons. Unique electrical properties have been predicted such as high electron mobility. ${ }^{2}$ Quantized conductances ${ }^{3}$ and improved transconductance in field effect transistors ${ }^{4}$ have been reported. The plasmon dispersion is a very sensitive probe for important physical parameters of these devices such as the electron density distribution and the electrostatic confinement potential. A classical description of the plasmon dispersion in wires has been proposed by Eliasson and co-workers. ${ }^{5}$ It applies when the lateral size is such that single electron quantization is negligible (>30 nm typically). The predicted dispersions have been successfully tested recently, ${ }^{6,7}$ on deep etched wires studied by Raman scattering. Experimental plasmon frequencies for other fundamental geometries such as circular $\operatorname{dots}^{8}$ and rings 9 could likewise be explained within the classical models. ${ }^{10,11}$ For more complicated lateral confinement geometries, the long-range character of the electron-electron interaction renders the numerical problem increasingly difficult. From the experimental side, the fabrication of such devices on a nanometer scale necessitates innovative steps in semiconductor process technology. The spectroscopy of such systems is thus very useful to further analyze the physics of the low-dimensional electron gas, in particular, to understand the influence of lateral boundaries on its electronic and optical properties, and to contribute to the assessment of their ultimate dimensions.

In this paper, we present an experimental and theoretical determination of the plasmon dispersion in wires with a strong periodic modulation of the lateral size along the wire axis. We describe a numerical solution for the plasmon dispersion in laterally confined $2 \mathrm{D}$ electron systems. Its predictions are in excellent agreement with the experimental dispersion, which we have measured by Raman scattering on deep etched modulation-doped GaAs quantum wells. We demonstrate the appearance of both extended and confined 1D plasmons and analyze the criteria for the existence of these two behaviors. The dispersion of extended 1D plasmons displays energy gaps at zone center and zone edge, reflecting the longitudinal modulation. The energies of con- fined 1D plasmons are significantly modified due to the longrange character of the Coulomb interaction.

We have fabricated modulated wires by deep reactive ion etching of a $\mathrm{GaAs} / \mathrm{Ga}_{x} \mathrm{Al}_{1-x} \mathrm{As}$ modulation-doped single quantum well, followed by an anodic oxidation to passivate the damaged part on the lateral surfaces of the wires. ${ }^{12}$ The initial sample and the process are identical to the one already described in Ref. 6 for the fabrication of unmodulated wires. We have realized arrays of 160 parallel, $160-\mu \mathrm{m}$-long periodically modulated wires. The period of the array $(1 \mu \mathrm{m})$ prevents any significant electrostatic coupling between the wires. Three different arrays have been realized in order to investigate both a change in the modulation amplitude at fixed period (samples $A$ and $B$ ) and a change in the modulation period with close modulation amplitude (samples $A$ and $C$ ). Figure 1 shows a scanning electron microscopy (SEM) photograph which illustrates the good definition of the modulated profile. We give in Table I for each sample the nominal parameters $a, b, c, d$ according to the definitions shown in Fig. 1. An accurate determination of these parameters from the SEM micrograph is difficult due to the complexity of the profile. The oxidation rate, which appears to be highly isotropic, has been previously calibrated on unmodulated wires. We have thus determined $a, b, c, d$ according to the method developed in Ref. 6 for unmodulated wires: the coded width, corrected from the oxide thickness $(48 \mathrm{~nm}$ on each surface).

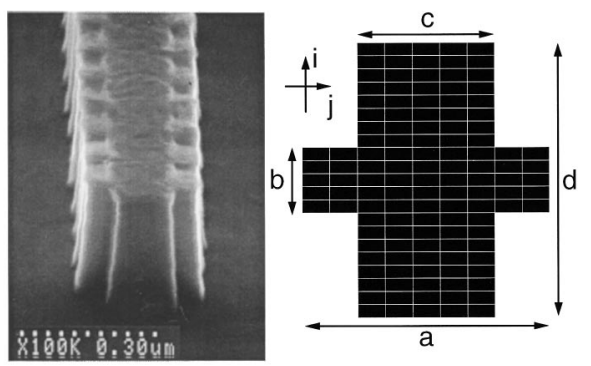

FIG. 1. (left) SEM micrograph of sample $A$, (right) schematic representation of the discretization grid and definition of the model parameters within the unit cell. 
TABLE I. Comparison between the nominal parameters of the three different samples studied in this work, and the one deduced from the fit of the plasmon dispersions to our classical model.

\begin{tabular}{|c|c|c|c|c|c|c|}
\hline \multicolumn{7}{|c|}{ Dimensions (nm) } \\
\hline & \multicolumn{2}{|c|}{ A } & \multicolumn{2}{|c|}{ B } & \multicolumn{2}{|c|}{$\mathrm{C}$} \\
\hline & nominal & electrical & nominal & electrical & nominal & electrical \\
\hline$d$ & 250 & 250 & 250 & 250 & 300 & 300 \\
\hline$a$ & 184 & 172 & 184 & 160 & 200 & 200 \\
\hline$c$ & 84 & 96 & 44 & 50 & 100 & 127 \\
\hline$b$ & 34 & 50 & 34 & 50 & 90 & 90 \\
\hline & \multicolumn{6}{|c|}{ Density $\left(10^{11} \mathrm{~cm}^{-2}\right)$} \\
\hline & \multicolumn{2}{|c|}{5.9} & \multicolumn{2}{|c|}{5.3} & \multicolumn{2}{|c|}{4} \\
\hline
\end{tabular}

Raman quasibackscattering experiments have been performed in pumped liquid Helium with an incident laser energy $(\sim 1.59 \mathrm{eV})$ in strong resonance with interband transitions associated to the second conduction subband of the GaAs single quantum well. The plasmon wave vector along the wire is varied either by tilting the angle of incidence along the wire axis or by rotating the sample around the normal to the sample surface, according to the following relation:

$$
q=\frac{2 \pi}{\lambda}\left(\sin \theta_{i}+\sin \theta_{s}\right) \cos \varphi
$$

where $\lambda$ is the incident wavelength, $\theta_{i}$ and $\theta_{s}$ the incidence angle of the incoming and scattered light, respectively, and $\varphi$ the angle between the plane of incidence and the wire axis. When $\varphi=0$, the parity with respect to wire axis is preserved, and even (respectively, odd) plasmons are observed in parallel and perpendicular polarization, respectively. ${ }^{13}$ For nonvanishing $\varphi$, all the modes are activated in both polarizations. These selection rules remain valid in the modulated wires due to the symmetry of the modulation. Polarized Raman spectra, obtained on samples $A$ and $C$ with $\varphi=0$, are compared in Fig. 2 with the spectra obtained on a $100-\mathrm{nm}-$ wide unmodulated wire in the same conditions. ${ }^{6}$ In the presence of the modulation, the lowest-energy even plasmon, allowed in this configuration, split into two components. The wave vector, where this splitting is minimum increases with decreasing longitudinal period and is given by $q=\pi / d$. From Raman experiments in other configurations, we find that the mode with the second-lowest energy (the lowest-energy odd mode) displays almost no dispersion. We attribute both observations to the effect of the periodic modulation of the lateral width.

In order to model these experimental results, we have linearized the plasmon equations in the classical approximation. ${ }^{5}$ The electron density fluctuation $n_{1}(x, y)$ at frequency $\omega$ and the associated fluctuation $\phi_{1}(x, y, z)$ of the electrostatic potential must satisfy the two following equations:

$\frac{\partial^{2} \phi_{1}}{\partial x^{2}}+\frac{\partial^{2} \phi_{1}}{\partial y^{2}}+\frac{\partial^{2} \phi_{1}}{\partial z^{2}}=\frac{e}{\varepsilon} n_{1} \delta(z), \quad$ (3D Poisson equation)

and

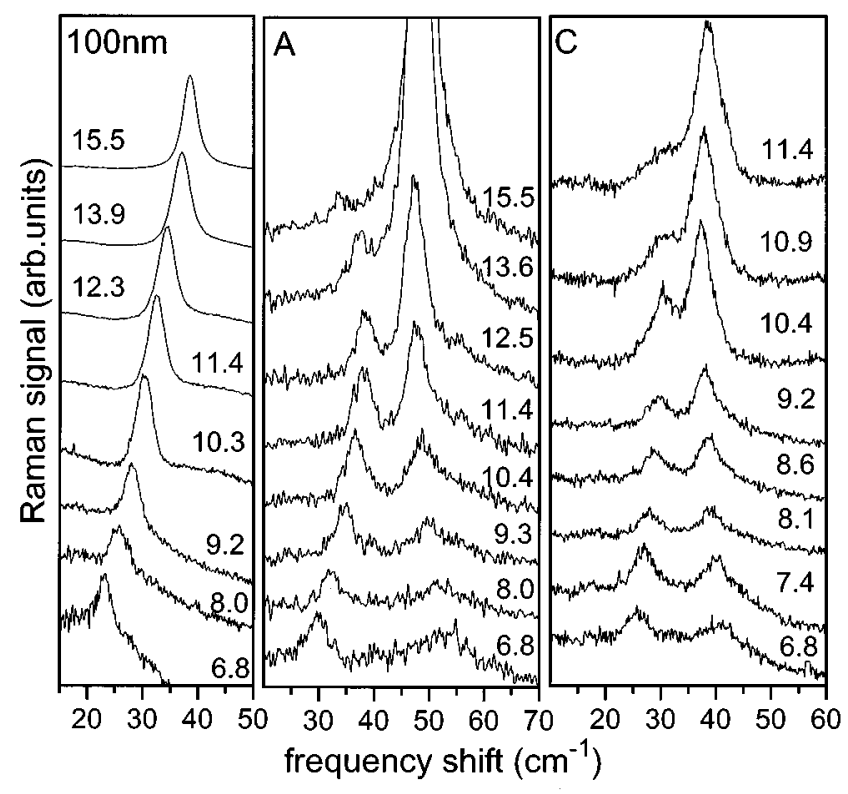

FIG. 2. Raman spectra obtained in parallel polarization on sample $A$, sample $C$, and a 100 -nm-wide unmodulated wire, for different values of the wave vector along the wire axis. This parameter is given in $\mu \mathrm{m}^{-1}$ for each spectrum in the figure.

$$
\omega^{2} n_{1}=\frac{e}{m^{*}}\left[\frac{\partial}{\partial x}\left(n_{0} \frac{\partial \phi_{1}}{\partial x}\right)+\frac{\partial}{\partial y}\left(n_{0} \frac{\partial \phi_{1}}{\partial y}\right)\right],
$$

(2D conservation equation),

where $n_{0}(x, y)$ is the equilibrium density distribution in the system. The electrons are assumed to be perfectly confined in the $z=0$ plane. The dielectric constant $\varepsilon$ and the effective mass $m^{*}$ are supposed to be constant over the whole system. Upon elimination of $n_{1}$ between both equations, one finds

$$
\begin{aligned}
& \omega^{2} \phi_{1}(\mathbf{r}, z=0) \\
& \quad=-\frac{e^{2}}{\varepsilon m^{*}} \iint \frac{d^{2} \mathbf{r}^{\prime}}{\left|\mathbf{r}-\mathbf{r}^{\prime}\right|} \nabla \cdot\left[n_{0}\left(\mathbf{r}^{\prime}\right) \nabla \phi_{1}\left(\mathbf{r}^{\prime}, z=0\right)\right] .
\end{aligned}
$$

The roots $\omega$ of this integrodifferential equation are the plasmon oscillations of the electron system. In absence of lateral confinement, $n_{0}$ becomes a constant and $\phi_{1}$ can be Fourier transformed in two dimensions. The integral in Eq. (1) can be performed analytically and one recovers the usual 2D plasmon dispersion. When lateral confinement is introduced, numerical solutions of this eigenvalue equation must be considered, in particular because Eq. (1) is strongly nonlocal with slowly decreasing long-range interactions. We have assumed abrupt boundary conditions: the equilibrium density $n_{0}$ is taken as a constant within the wires and vanishes outside. We have used a finite differences technique to determine the plasmon dispersion in periodically modulated wires and projected the integrodifferential equation (1) over a basis of local functions constant on each rectangle $i j$ of the grid as shown schematically in Fig. 1. The geometrical parameters of the model are shown in the figure and the resulting discrete set of linear equations writes 


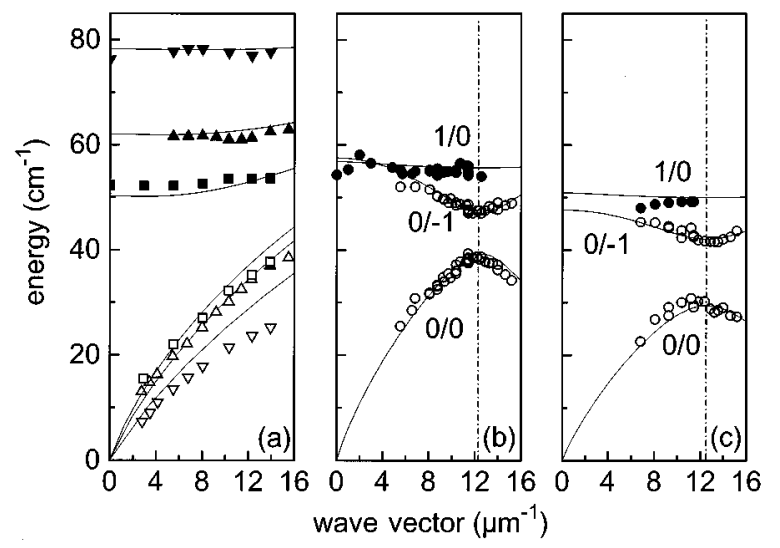

FIG. 3. Comparison between experimental and theoretical plasmon dispersions along the wire axis for mode 0 (open symbols) and mode 1 (closed symbols) in (a) three unmodulated wires with different widths: 60 (down triangles), 100 (up triangles), and $180 \mathrm{~nm}$ (squares) and in two different modulated wires: (b) sample $A$ and (c) sample $B$.

$$
\begin{aligned}
\omega^{2} \phi_{i j}= & -\frac{e^{2} n_{0}}{\varepsilon m^{*}} \sum_{k, l} C(q, i-k, j-l)\left[\left(2 \varphi_{k l}-\varphi_{k+1 l}\right.\right. \\
& \left.\left.-\varphi_{k-1 l}\right) \frac{1}{\delta_{b}^{2}}+\left(2 \varphi_{k l}-\varphi_{k l+1}-\varphi_{k l-1}\right) \frac{1}{\delta_{a}^{2}}\right],
\end{aligned}
$$

where $\delta_{a}$ and $\delta_{b}$ are the lateral sizes of the unit rectangle.

The first factor $C(q, i-k, j-l)$ of each term is the effective Coulomb interaction between site $i j$ and site $k l$ plus all other sites equivalent to $k l$ by a periodic translation $n d$ along the wire. $q$ is the Bloch wave vector along this direction. This factor writes

$$
\begin{aligned}
& C(q, m, p) \\
& \quad=\sum_{n} e^{i q n d} \int_{-\delta_{a} / 2}^{\delta_{a} / 2} \int_{-\delta_{b} / 2}^{\delta_{b} / 2} \frac{d x d y}{\sqrt{\left(x-m \delta_{a}\right)^{2}+\left(y-p \delta_{b}-n d\right)^{2}}},
\end{aligned}
$$

where the integral can be analytically determined.

The second factor results from the projection of $\nabla \cdot\left(n_{0} \nabla \phi\right)$ on the local functions. The expression given in Eq. (2) is valid for any internal point of the lattice where $n_{0}$ is constant. For boundary points, $n_{0}$ is vanishing on one side of the points (two sides for a corner point). In this case, only the internal part of the discretized second derivative is retained and the corresponding term writes: $\phi_{i}-\phi_{i \pm 1}$ instead of $2 \phi_{i}-\phi_{i+1}-\phi_{i-1}$, where $i$ is a line or column index and only $i+1$ or $i-1$ is considered, depending on the location of the point on the boundary.

The above theory quantitatively reproduces the dispersion of the three lowest branches on all three samples investigated. This is illustrated in Figs. 3(b) and 3(c) where we show the results for samples $A$ and $B$. In Fig. 3(a), we show for comparison the dispersion of the lowest even $(n=0)$ and the lowest odd $(n=1)$ modes in unmodulated wires with widths of 60,100 , and $180 \mathrm{~nm}$, which approximately correspond to the different widths entering the geometry of the modulated wires. Owing to the simplification of the unit cell with respect to the real one, we expect to obtain the best description for the lowest plasmon branches, which are

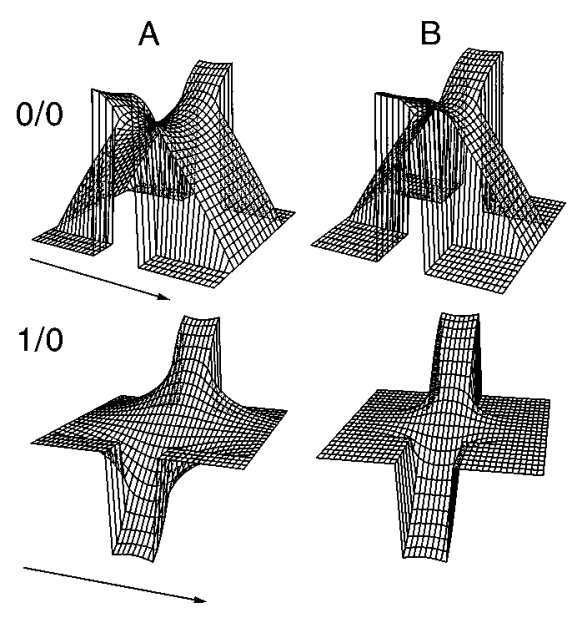

FIG. 4. Fluctuations of the electrostatic potential associated with the folded plasmon mode $0 / 0$ and the confined one $1 / 0$, calculated at zone edge for samples $A$ and $B$, respectively. The arrows indicate the wire axis.

mostly sensitive to the lowest Fourier components of the profile. Indeed, we have obtained an excellent fit of the dispersion curves, using model parameters (see Table I) close to the nominal ones. The modes in the modulated wires are labelled with two indices $n / m$, the first of which corresponds to the lateral confinement perpendicular to the wire axis, and the second of which is connected to umklapp processes due to the longitudinal modulation. The gap opening at zone edge, due to the anticrossing between mode $0 / 0$ and $0 /-1$, strongly increases when the modulation is deepened. On the other hand, the relative positions of mode $0 /-1$ and $1 / 0$, as well as their dispersion, are very sensitive to the exact width of the wide part of the wire, in which mode $1 / 0$ is confined (see below). As shown in Table I, we have slightly adjusted $a, b$, and $c$ with respect to the nominal values in order to improve the agreement with experiment. These variations are attributed to uncertainties in the oxidation depth with respect to its determination on unmodulated wires, and to the simplification of the model geometry with respect to the real profiles. A solution of the plasmon dispersion in confined electron systems with arbitrary geometries, based on finite elements technique, using a triangular mesh, is presently under development. Results for realistic profiles will be presented elsewhere.

In order to better understand the impact of the lateral modulation on the plasmon dispersion curves, we will now discuss the calculated electrostatic potential associated to the observed branches. They are shown in Fig. 4 at the longitudinal zone edge for branch $n / m=0 / 0$ and $1 / 0$ in the unit cells corresponding to samples $A$ and $B$. The potential associated to mode $0 / 0$ is fully delocalized within the whole unit cell. We attribute this property to the overlap between the energy bands of mode $n=0$ in the unmodulated wires with lateral widths $a$ and $c$, respectively. The corresponding dispersion curve displays a folding behavior with gap openings at zone center and zone edge. On the contrary, mode $1 / 0$ is confined in the widest part of the unit cell. The lowest energy of the odd plasmon $n=1$ indeed is smaller in the wide constituting wire than in the narrow one. As plasmon 1 is not coupled to 
plasmon 0 at the same energy, due to their opposite parities, an energy range appears where no allowed modes are available in the narrow section. Plasmon confinement thus takes place. Mode 1/0 exhibits this behavior in the two considered samples. The corresponding dispersions are very flat. The existence of higher confined plasmons associated to branch 1 depends on the ratio of lateral sizes $a$ and $c$. Moreover, the analysis of higher branches becomes more intricate because of the strong interbranch coupling, for instance between modes 0 and 2, due to the longitudinal modulation.

The coexistence of folded and confined excitations has been previously discussed for other collective excitations such as for instance phonons in superlattices. ${ }^{14}$ There, however, the modulation of the sound velocity, leading to the folding effect is limited in amplitude. The modulation of the lateral width which is employed here can be arbitrary large. It should also allow the continuous transition towards confined plasmons in boxes to be studied. Moreover, the longrange character of the Coulomb interaction produces some specific behavior in the quantitative description of the energies of the modulated plasmons. For instance, in some cases, the energy of the confined mode $1 / 0$ is not increasing but decreasing when the width of the constriction is reduced though this reduction induces an increasing confinement as can be seen in Fig. 4. The energy of mode $1 / 0$ can be even smaller than the one of mode 1 in the wire without constriction. This behavior indeed is in contradiction to the usual trend for excitations with increasing dispersion curves, that a stronger confinement induces increasing energy. We have understood that this behavior results from the associated reduction of the average number of "neighbors" interacting with any site inside the wide part. This implies a significant decrease of the total Coulomb force on the site and thereby, of the plasmon energies. This effect overcompensates the increase due to the confinement. The same explanation applies to the decrease of the energy of the lowest 1D plasmon $(n=0)$ in unmodulated wires, contrary to the higher ones $(n \geqslant 1)$, when the lateral width is reduced. ${ }^{6}$

In conclusion, we have reported in this paper a quantitative determination of plasmons in doped wires, the lateral width of which is periodically modulated. The results presented here demonstrate the coexistence of longitudinally confined and extended plasmons in 1D systems with modulated boundary conditions. Within a classical frame, we have reproduced well the experimental dispersions, including gap openings at zone edge, deduced from Raman scattering on deep etched wires. The excellent ability of our theoretical approach, assuming a constant equilibrium density over the whole wire, to reproduce the dispersion of electronic excitations in unmodulated ${ }^{6}$ and strongly modulated wires suggests that nearly abrupt boundary conditions apply to oxidized GaAs lateral surfaces, at least at low temperature under illumination. The latter and the observation of large gaps in one-dimensional plasmon dispersion show that our deep RIE process is a suitable method to define complex geometries on a very low scale $(10 \mathrm{~nm})$ while preserving the high quality of the initial 2D electron gas. This offers a powerful tool to investigate new physical problems in low-dimensional systems with almost arbitrary geometries, for instance the continuous transition from quantum wires to quantum boxes.

It is a pleasure for us to thank C. Mayeux, D. Arquey, and C. Dupuy for expert technical assistance and J. M. Gerard and J. Y. Marzin for useful discussions and a critical reading of the manuscript. We gratefully acknowledge financial support from the European Union (Human Capability and Mobility program).
*Present address: Siemens AG, Semiconductor Division, OttoHahn-Ring 6, 81739 Munich, Germany.

${ }^{1}$ For a review, see the Proceedings of the Sixth International Conference on Modulated Semiconductor Structures [Solid-State Electron. 37, 523-1344 (1994)] and Proceedings of the Seventh International Conference on Modulated Structures (Solid-State Electron.) (to be published).

${ }^{2}$ H. Sakaki, Jpn. J. Appl. Phys. 19, L735 (1980).

${ }^{3}$ D. A. Wharam, T. J. Thornton, R. Newbury, M. Pepper, H. Ahmed, J. E. F. Frost, D. G. Hasko, D. C. Peacok, D. A. Ritchie, and G. A. C. Jones, J. Phys. C 21, L209 (1988); B. J. van Wees, H. van Houten, C. W. J. Beenaker, J. G. Williamson, L. P. Kouwenhoven, D. van der Marel, and C. T. Foxon, Phys. Rev. Lett. 60, 848 (1988).

${ }^{4}$ S. Bollaert, P. Legris, E. Delos, A. Cappy, P. Debray, and J. Blanchet, IEEE Electron Device Lett. 41, 1716 (1994).

${ }^{5}$ G. Eliasson, J. W. Wu, P. Hawrylak, and J. J. Quinn, Solid State Commun. 60, 41 (1986).
${ }^{6}$ C. Dahl, B. Jusserand, and B. Etienne, Solid-State Electron. 40, 261 (1996).

${ }^{7}$ T. Egeler, G. Abstreiter, G. Weimann, T. Demel, D. Heitmann, P. Grambow, and W. Schlapp, Phys. Rev. Lett. 65, 1804 (1990).

${ }^{8}$ S. J. Allen, Jr., H. L. Störmer, and J. C. Hwang, Phys. Rev. B 28, 4875 (1983).

${ }^{9}$ C. Dahl, J. P. Kotthaus, H. Nickel, and W. Schlapp, Phys. Rev. B 48, 15480 (1993).

${ }^{10}$ A. L. Fetter, Phys. Rev. B 33, 5221 (1986).

${ }^{11}$ H. L. Cui, V. Fessatidis, and O. Kühn, Superlattices Microstruct. 17, 173 (1995).

${ }^{12}$ J. Y. Marzin, A. Izrael, and L. Birotheau, Solid-State Electron. 37, 1091 (1994).

${ }^{13}$ C. Dahl, B. Jusserand, and B. Etienne, Phys. Rev. B 51, 17211 (1995).

${ }^{14}$ B. Jusserand and M. Cardona, in Light Scattering in Solids V, edited by M. Cardona and G. Güntherodt (Springer, Berlin, 1989). 